\title{
Bandwidth Efficient Spatial Modulation by Signalling in the Power Domain
}

\author{
Christos Masouros†, Member, IEEE, Lajos Hanzoł, Fellow, IEEE \\ $\dagger$ Dept. of Electrical \& Electronic Eng., University College London, London, UK \\ $\ddagger$ School of Electronics and Computer Science, University of Southampton, UK \\ Email: chris.masouros@ieee.org, lh@ecs.soton.ac.uk
}

\begin{abstract}
We explore a bandwidth efficient transmission scheme that amalgamates multiple-input-multiple-output spatial multiplexing (SMX) with receive antenna based spatial modulation (RSM). The RSM here is applied to the combined spatial and power-level domain, not by activating and de-activating the receive antennas, but rather by choosing between two power levels $\left\{P_{1}, P_{2}\right\}$ for the received symbols in these antennas, such that all receive antennas are active and SMX can still be accommodated. This allows for the coexistence of RSM with SMX and the results show an increased bandwidth efficiency for the proposed scheme compared to both SMX and RSM. We further carry out a mathematical analysis to optimize the ratio between $P_{1}$ and $P_{2}$ for attaining the minimum error rates. Our analytical and simulation results demonstrate significant bandwidth efficiency gains for the proposed scheme compared to conventional SMX and RSM.
\end{abstract}

Index Terms-Spatial modulation, spatial multiplexing, multiple-input-multiple-output systems, transmit precoding

\section{INTRODUCTION}

Spatial Modulation (SM) has been conceived for implicitly encoding information in the index of the specific antenna activated for the transmission of the modulated symbols, offering a low-complexity design alternative [1]. Its central benefits include the absence of inter-antenna interference (IAI) and the fact that it only requires a subset (down to one) of Radio Frequency (RF) chains compared to spatial multiplexing. Accordingly, the inter-antenna synchronization is also relaxed. Early work has focused on the design of receiver algorithms for minimizing the bit error ratio (BER) of SM at a low complexity [1]-[7]. In addition to receive processing, recent work has also proposed constellation shaping for SM [8]-[18].

Closely related work has focused on applying the concept of SM to the receive antennas (RAs) of the communication link, forming the RA-based spatial modulation (RSM) concept [19]. By means of transmit precodng (TPC) [20]-[23], this technique targets a specific subset of RAs which receive information symbols, while the rest of the RAs receive only noise. This may be achieved by using zero forcing (ZF)-TPC and transmitting a combination of information symbols and zeros to the RAs depending on the spatial symbols to convey. As opposed to conventional SM where a subset of RF chains is deployed, here all TAs and RAs are active and therefore there are no RF chain reductions. Still, the computational complexity of the receivers is drastically reduced where, simply the indices of the targeted RAs have to be detected and the classic symbols received at the activated RAs are then demodulated.
Inspired by the above RSM philosophy, here we propose a dual-layered transmission scheme, which intrinsically amalgamates a full spatial multiplexing (SMX) with RSM. Firstly, we note that since for RSM all TAs and RAs are active, there are no RF chain reductions and this motivates the full SMX approach. To accommodate the SMX, we apply a SM to the combined spatial and receive-power domain, where instead of sending a combination of information symbols and zero power to the RAs, we apply two different power levels for distinguishing between the 'active' and 'inactive' RAs. In this manner, the spatial symbols are formed based on the power levels detected. We demonstrate that this improves the bandwidth efficiency with respect to SMX and RSM. We further develop a pairwise error probability (PEP) analysis for the proposed scheme and use this to analytically derive the optimum power ratio between the two sets of antennas that carry the spatial symbol for the proposed scheme, for minimizing the probability of detection errors.

While this work focuses on a single-link scenario, the proposed technique can be readily extended to a multiuser DL scenario, where the dual-layered transmission and the related RA-based spatial modulation take place on a per-user basis, as facilitated by the ZF-TPC employed at the base station (BS). Note that the proposed scheme does not consist of a power allocation scheme in the sense of allocating power according to the Quality of Service (QoS) requirements of the user. This power allocation may be applied in addition to the proposed scheme in the multi-user scenario, where different users with different QoS requirements employ different sets of powers $\left\{P_{1}, P_{2}\right\}$ accordingly.

\section{SYSTEM MOdEL AND RSM}

Consider a MIMO system, where the transmitter and receiver are equipped with $N_{t}$ and $N_{r}$ antennas, respectively. For simplicity, unless stated otherwise, in this paper we assume that the transmit power budget is limited as $P=1$. For the case of the closed-form TPCs of [20]-[23], it is required that $N_{t} \geq N_{r}$. The above channel is modeled by the equation

$$
\mathbf{y}=\mathbf{H t}+\mathbf{w}
$$

where $\mathbf{y}$ is the vector of received symbols in all receive antennas and $\mathbf{H}$ is the MIMO channel vector with elements $h_{m, n}$ representing the complex channel coefficient between the $n$-th TA and the $m$-th RA. Furthermore, $\mathbf{t}$ is the vector 
of precoded transmit symbols that will be discussed in the following and $\mathbf{w} \sim \mathcal{C N}\left(0, \sigma^{2} \mathbf{I}\right)$ is the additive white Gaussian noise (AWGN) component at the receiver.

RSM targets a subset of the RAs by sending information symbols to these RAs and zero power to the rest of the RAs. While for RSM all RAs have to be on to detect the arrival of information symbols, for coherence with the SM literature we shall refer to the antennas as 'active' and 'inactive' depending on whether they do or do not receive information symbols, respectively. The specific combination of RAs that do receive symbols implicitly conveys the symbol transmitted in the spatial domain. The above RA subset transmission is achieved by forming a super-symbol vector in the form $\mathbf{s}_{m}^{k}=\mathbf{e}_{k} b_{m}=\left[0, \ldots, b_{m_{1}}, \ldots, 0, \ldots, b_{m_{2}}, \ldots, 0\right]^{T}$ with $N_{a}$ non-zero elements, where $\mathbf{e}_{k}$ is a diagonal matrix of size $N_{r}$ with elements taken from the set $\{1,0\}$ on its diagonal, that represents the RAs that are activated. The notation $[.]^{T}$ denotes the transpose operator. Here, $b_{m_{i}}, m_{i} \in\{1, \ldots, M\}$ is a symbol taken form an $M$-order modulation alphabet that represents the transmitted waveform in the baseband domain conveying $\log _{2}(M)$ bits and $k$ represents the index of the $N_{a}$ activated RAs (the index of the non-zero elements in $\mathbf{s}_{m}^{k}$ ) conveying $\log _{2}\left(\begin{array}{l}N_{r} \\ N_{a}\end{array}\right)$ bits in the spatial domain. Accordingly, the total number of bits conveyed per super-symbol for RSM is $\beta=N_{a} \log _{2}(M)+\log _{2}\left(\begin{array}{l}N_{r} \\ N_{a}\end{array}\right)$. The transmitter then sends

$$
\mathbf{t}=f \mathbf{T} \mathbf{s}_{m}^{k}
$$

where $\mathbf{T}=\mathbf{H}^{H}\left(\mathbf{H} \mathbf{H}^{H}\right)^{-1}$ is the zero forcing TPC [20] that preserves the form of $\mathbf{s}_{m}^{k}$ at the receiver. The factor $f=\sqrt{\frac{1}{\operatorname{tr}\left(\mathbf{T} \mathbf{T}^{H}\right)}}$, where $\operatorname{tr}($.$) denotes the trace operator,$ normalizes the average transmit power to $P=1$. The received symbol vector can be written as

$$
\mathbf{y}=f \mathbf{H} \mathbf{T} \mathbf{s}_{m}^{k}+\mathbf{w}=f \mathbf{s}_{m}^{k}+\mathbf{w},
$$

where clearly all IAI is removed. At the receiver, a joint maximum likelihood (ML) detection of both the RA index and the transmit symbol is obtained by the minimization

$$
\begin{aligned}
{\left[\hat{s}_{m}, \hat{k}\right] } & =\arg \min _{i}\left\|\mathbf{y}-\dot{\mathbf{y}}_{i}\right\| \\
& =\arg \min _{m_{i}, k_{i}}\left\|\mathbf{y}-f \mathbf{H T} \mathbf{T s}_{m_{i}}^{k_{i}}\right\|,
\end{aligned}
$$

where $\|\mathbf{x}\|$ denotes the norm of vector $\mathbf{x}$ and $\dot{\mathbf{y}}_{i}$ is the $i$-th constellation point in the received SM constellation. A lowcomplexity decoupled approach is also proposed in [19] where first the active antenna indices are detected in the form of

$$
\hat{k}=\arg \max _{j \in \mathcal{J}} \sum_{i=1}^{N_{a}}\left|y_{j, i}\right|^{2},
$$

where $\mathcal{J}$ denotes the set of symbols in the spatial domain, and then the classic modulated symbols are detected by

$$
\hat{b}_{m_{i}}=\arg \min _{n_{i} \in \mathcal{Q}}\left|y_{\hat{k}, i} / f-b_{n_{i}}\right|^{2},
$$

where $\mathcal{Q}$ denotes the modulation constellation and $b_{n_{i}}$ are the symbols in the modulated symbol alphabet. For reasons of computational complexity, we shall focus on the latter detector in this work.

\section{PRoposed DUAL-LAYERED TRANSMISSION (DLT)}

From the above system description, it can be seen that for the particular case of RSM, while the detection complexity is clearly reduced with respect to SMX, there are no savings in RF complexity, since all $N_{r}$ RAs have to be activated and receiving for the detection in (5)-(6). Still, by forming a subset of beams towards the receiver, the bandwidth efficiency, i.e. the number of bits per channel use, is generally lower for RSM than for SMX. Motivated by this, we propose a dual layered approach combining SMX with RSM, where the bandwidth efficiency of conventional SMX MIMO transmission is strictly enhanced by encoding spatial bits in the RSM fashion in the received power domain, by selecting two distinct, non-zero power levels for the transmitted super-symbols instead of the 'on-off' RSM transmission in the $\{1,0\}$ manner. This allows for non-zero elements throughout the super-symbol vector $\mathbf{s}_{m}^{k}$, hence supporting a full SMX transmission in the modulated signal domain. The block diagram of the proposed scheme is shown in Fig.1.

1) Transmitter: Here, we employ a full data vector in the form of $\mathbf{b}_{m}=\left[b_{m_{1}}, b_{m_{2}} \ldots, b_{m_{N_{r}}}\right]^{T}$, with all elements being non-zero, and the encoding of the spatial bits is achieved by allocating different powers to the received symbols according to the spatial symbol $k$, by applying the power diagonal allocation matrix $\mathbf{P}_{k}$

$$
\mathbf{s}_{m}^{k}=\mathbf{P}_{k} \mathbf{b}_{m}=\left[s_{m_{1}}, s_{m_{2}} \ldots, s_{m_{N_{r}}}\right]^{T},
$$

with elements $p_{i}, i \in\left[1, N_{r}\right]$ are taken from the set $\left\{P_{1}, P_{2}\right\}$ according to the spatial symbol $k$. Note that classic QoS based power allocation can be applied in addition to this process by employing an additional power allocation matrix on top of $\mathbf{P}_{k}$. The receiver can then remove this additional matrix by simple inversion, in order to detect the spatial symbol. For notational simplicity and to keep the focus of the discussion on the proposed concept, we neglect QoS-based power allocation.

2) Receiver: At the receiver side the explicit knowledge of the power levels $\left\{P_{1}, P_{2}\right\}$ is not required, as long as the detector can distinguish between the two power levels. The received signal of (3) can be decomposed as

$$
\begin{aligned}
& y_{p}=f \sqrt{P_{1}} b_{m_{p}}+w_{p}, p \in \mathcal{A}, \\
& y_{q}=f \sqrt{P_{2}} b_{m_{q}}+w_{q}, q \in \mathcal{I},
\end{aligned}
$$

where $\mathcal{A}$ and $\mathcal{I}$ denote the sets of 'active' and inactive' antennas respectively. Hence, the receive processing is similar to the conceived one for RSM, with the difference that the classic modulated symbols of all RAs have to be detected, as opposed to those of $N_{a}$ antennas only for RSM. Accordingly, the receiver first detects the set of antennas with the highest received powers and then detects the classic modulated symbols at all RAs according to

$$
\hat{k}=\arg \max _{j \in \mathcal{J}} \sum_{i=1}^{N_{a}}\left|y_{j, i}\right|^{2},
$$

where $\mathcal{J}$ denotes the set of symbols in the spatial domain, and

$$
\hat{\mathbf{b}}_{m}=\arg \min _{n \in \mathcal{Q}}\left|\mathbf{y} / f-\mathbf{b}_{n}\right|^{2},
$$



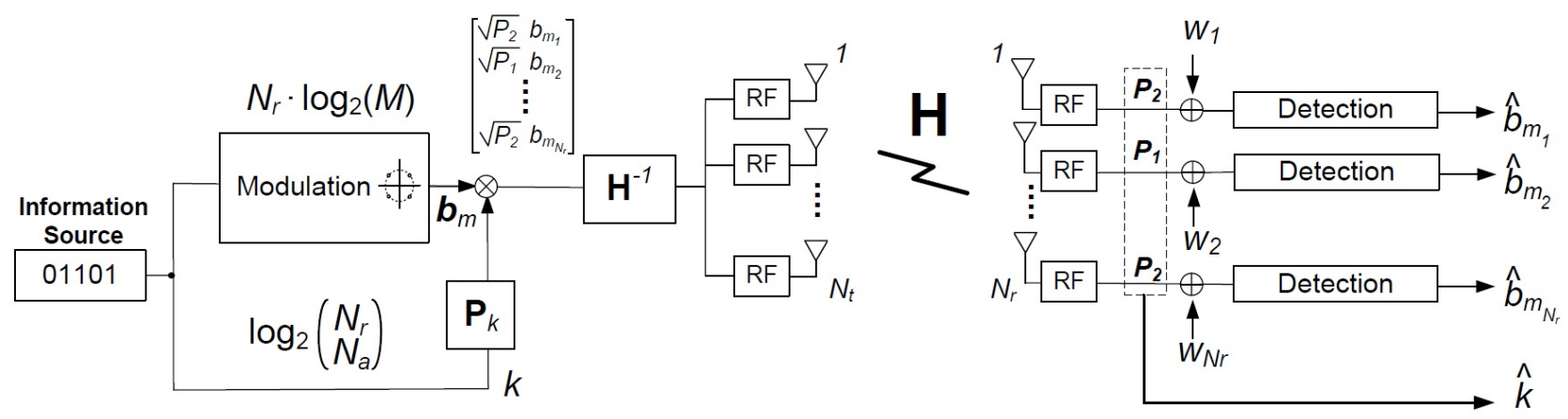

Fig. 1. Block diagram of DLT transmission.

\begin{tabular}{|l|r|}
\hline SMX & $\beta=N_{r} \log _{2}(M)$ \\
\hline RSM & $\beta=N_{a} \log _{2}(M)+\log _{2}\left(\begin{array}{l}N_{r} \\
N_{a}\end{array}\right)$ \\
\hline DLT & $\beta=N_{r} \log _{2}(M)+\log _{2}\left(\begin{array}{l}N_{r} \\
N_{a}\end{array}\right)$ \\
\hline
\end{tabular}

TABLE I: Bandwidth Efficiency in bits per channel use for SMX, RSM and DLT.

where $\mathcal{Q}$ denotes the classic modulation constellation and $b_{n}$ are the symbols in the modulated symbol alphabet.

Clearly, the encoding process in (7) encodes $N_{r} \log _{2}(M)$ bits in the modulated symbol domain and an additional $\log _{2}\left(\begin{array}{l}N_{r} \\ N_{a}\end{array}\right)$ bits in the spatial domain. This results in a total of

$$
\beta=N_{r} \log _{2}(M)+\log _{2}\left(\begin{array}{l}
N_{r} \\
N_{a}
\end{array}\right)
$$

bits per transmitted super-symbol for DLT, which is strictly greater than that for SMX and RSM. Here, the notation $N_{a}$ denotes the number of antennas receiving symbols at the power level $P_{1}$. We should emphasize that, even though all RAs are active for both RSM and the proposed DLT, for coherence with the SM literature, we shall adhere to the terms 'active' and 'inactive' to indicate the antennas receiving $\{1,0\}$ and $\left\{P_{1}, P_{2}\right\}$ for RSM and DLT respectively. A comparison of the bandwidth efficiencies of SMX, RSM and DLT is shown in Table I, where it can be seen that the proposed DLT approach has an improved BE compared to the conventional approaches.

\section{A. Symbol Power Levels}

As regards to the resulting bit error ratio (BER) performance, the set of spatial powers $\left\{P_{1}, P_{2}\right\}$ must be carefully selected so that there is sufficient separation between the two power levels $P_{1}, P_{2}$ for correct detection of the 'active' antennas, and hence the spatial symbol $k$, in the presence of noise. Still,the symbols received with $P_{2}<P_{1}$, that dominate the BER of the modulated symbol detection, must experience a sufficiently high signal to noise ratio (SNR) that is adequate for reliable demodulation. Let us therefore define the power ratio

$$
\alpha=\frac{P_{2}}{P_{1}}
$$

as the ratio between the two power levels transmitted, which is optimized in the following results. Since $N_{a}$ symbols are transmitted with power $P_{1}$ and the remaining $N_{r}-N_{a}$ symbols have a power of $P_{2}$, given a total power budget of $P=1$, we have

$$
P_{1}=\frac{1}{\left(N_{r}-N_{a}\right) \alpha+N_{a}}, P_{2}=\frac{\alpha}{\left(N_{r}-N_{a}\right) \alpha+N_{a}} .
$$

Clearly, since the power levels $P_{1}, P_{2}$ influence the reliability of detection for the modulated symbols and since the ratio $\alpha$ determines the detection reliability of the spatial symbols, $\alpha$ can be optimized for best BER performance. In the following, we derive a closed-form expression for the optimum $\alpha$ value for an $M$-order PSK modulation, where it can be seen that this optimum value is independent of both $N_{r}$ and of $N_{a}$.

\section{PEP ANAlysis AND Optimum POWER RATIO $\alpha$}

In this section we carry out a PEP analysis for the proposed DLT scheme by deriving the PEP between the pair of symbols $\mathbf{s}_{m}^{k}$ and $\mathbf{s}_{n}^{l}$ in the super-imposed spatial and classic modulation constellations, following the analysis in [19]. Accordingly, we define the PEP as $\mathcal{P}\left(\mathbf{s}_{m}^{k} \rightarrow \mathbf{s}_{n}^{l}\right)$ and use the union-bound for the average bit error probability $P_{e}$, which is expressed as

$$
P_{e} \leq \frac{1}{b} E\left\{\sum_{\mathbf{s}_{m}^{k} \in \mathcal{B}} \sum_{\mathbf{s}_{n}^{l} \in \mathcal{B} \neq \mathbf{s}_{m}^{k}} d\left(\mathbf{s}_{m}^{k}, \mathbf{s}_{n}^{l}\right) \mathcal{P}\left(\mathbf{s}_{m}^{k} \rightarrow \mathbf{s}_{n}^{l}\right)\right\},
$$

where $d\left(\mathbf{s}_{m}^{k}, \mathbf{s}_{n}^{l}\right)$ is the Hamming distance between the bit representations of symbols $\mathbf{s}_{m}^{k}, \mathbf{s}_{n}^{l}$ and $\mathcal{B}=\mathcal{J} \cup \mathcal{Q}$ is the super-symbol constellation defined as the union of the spatial domain constellation and the classic modulation constellation. We have used the operator $\cup$ to define the union of sets. For the PEP we have the following theorem

Theorem 1: The PEP $\mathcal{P}\left(\mathbf{s}_{m}^{k} \rightarrow \mathbf{s}_{n}^{l}\right)$ for DLT can be expressed as

$$
\mathcal{P}\left(\mathbf{s}_{m}^{k} \rightarrow \mathbf{s}_{n}^{l}\right)=Q\left(\frac{f}{\sqrt{N_{0}}}\left(1-\sum_{i=1}^{N_{r}} \sqrt{p_{k_{i}} p_{l_{i}}} \mathcal{R}\left\{b_{m_{i}}^{*} b_{n_{i}}\right\}\right)\right),
$$

where $Q($.$) denotes the Gaussian q-function [24], \mathcal{R}\{$.$\} de-$ notes the real part of a number, $(.)^{*}$ denotes the complex conjugate operation and $N_{0}=2 \sigma^{2}$ is the noise power spectral density.

Proof: Let us first define $\mathbf{r}=\mathbf{y} / f$ and $\mathbf{v}=\mathbf{w} / f$ for use in the following expressions. The PEP of the super-symbol constellation can be expressed as 


$$
\begin{aligned}
\mathcal{P}\left(\mathbf{s}_{m}^{k} \rightarrow \mathbf{s}_{n}^{l}\right)= & \mathcal{P}\left(\left\|\mathbf{r}-\mathbf{s}_{m}^{k}\right\|^{2}>\left\|\mathbf{r}-\mathbf{s}_{n}^{l}\right\|^{2}\right) \\
= & \mathcal{P}\left(\sum_{i=1}^{N_{r}} p_{k_{i}}\left|b_{m_{i}}\right|^{2}-2 \mathcal{R}\left\{r_{i}^{*} \sqrt{p_{k_{i}}} b_{m_{i}}\right\}>\right. \\
& \left.\sum_{i=1}^{N_{r}} p_{l_{i}}\left|b_{n_{i}}\right|^{2}-2 \mathcal{R}\left\{r_{i}^{*} \sqrt{p_{l_{i}}} b_{n_{i}}\right\}\right)
\end{aligned}
$$

Since for PSK signals we have $\left|b_{m_{i}}\right|=1$, and $\sum_{i=1}^{N_{r}} p_{l_{i}}=$ $\sum_{i=1}^{N_{r}} p_{k_{i}}=1$ and $r_{i}=\sqrt{p_{k_{i}}} b_{m_{i}}+v_{i}$, by rearranging the terms in the probability expression, eq. (17) can be further simplified as

$$
\begin{aligned}
\mathcal{P}\left(\mathbf{s}_{m}^{k} \rightarrow \mathbf{s}_{n}^{l}\right) & =\mathcal{P}\left(\sum_{i=1}^{N_{r}} \mathcal{R}\left\{v_{i}^{*}\left(\sqrt{p_{l_{i}}} b_{n_{i}}-\sqrt{p_{k_{i}}} b_{m_{i}}\right)\right\}>\right. \\
1 & \left.-\sum_{i=1}^{N_{r}} \sqrt{p_{k_{i}} p_{l_{i}}} \mathcal{R}\left\{b_{m_{i}}^{*} b_{n_{i}}\right\}\right) .
\end{aligned}
$$

Let us define the random variable $\chi \hat{=} \sum_{i=1}^{N_{r}} \mathcal{R}\left\{v_{i}^{*}\left(\sqrt{p_{l_{i}}} b_{n_{i}}-\sqrt{p_{k_{i}}} b_{m_{i}}\right)\right\} \quad$ for which we have $\chi \in \mathcal{N}\left(0, A N_{0} / f^{2}\right)$ with

$$
A=\frac{\sum_{i=1}^{N_{r}} p_{l_{i}}\left|b_{n_{i}}\right|^{2}+p_{k_{i}}\left|b_{n_{i}}\right|^{2}}{2}=\frac{1}{2} \sum_{i=1}^{N_{r}} p_{l_{i}}+p_{k_{i}} .
$$

For the unity transmit power assumed in this paper it can be seen from (19) that $A=1$. Accordingly, for the PEP we have

$$
\mathcal{P}\left(\mathbf{s}_{m}^{k} \rightarrow \mathbf{s}_{n}^{l}\right)=\mathcal{P}\left(\chi>1-\sum_{i=1}^{N_{r}} \sqrt{p_{k_{i}} p_{l_{i}}} \mathcal{R}\left\{b_{m_{i}}^{*} b_{n_{i}}\right\}\right),
$$

which, for $\chi \in \mathcal{N}\left(0, N_{0} / f^{2}\right)$, leads to (16).

\section{A. Optimum Power Ratio $\alpha$}

As mentioned above, the power ratio $\alpha$ determines the reliability of detection for the spatial symbol, while the lower power level $P_{2}$ dominates the BER performance of the classic modulated symbols' detection. As the probability of error in (15) is dominated by the maximum PEP, the optimum power ratio should be selected as

$$
\alpha_{\text {opt }}=\arg \min _{\alpha} \max _{\mathbf{s}_{m}^{k}, \mathbf{s}_{n}^{l}}\left\{\mathcal{P}\left(\mathbf{s}_{m}^{k} \rightarrow \mathbf{s}_{n}^{l}\right)\right\} .
$$

To simplify the analysis, we shall treat the errors in the spatial and classic modulated symbols separately. Accordingly, for the maximum PEP $\mathcal{P}_{m}\left(s_{m_{i}}^{k} \rightarrow s_{m_{i}}^{l}\right)$ in the spatial domain only, we have the following Lemma

Theorem 2: The maximum PEP $\mathcal{P}_{m}\left(s_{m_{i}}^{k} \rightarrow s_{m_{i}}^{l}\right)$ for the spatial symbols in DLT can be expressed as

$$
\mathcal{P}_{m}\left(s_{m_{i}}^{k} \rightarrow s_{m_{i}}^{l}\right)=Q\left(\frac{f}{\sqrt{N_{0}}} \cdot \frac{\sqrt{P_{2}}-\sqrt{P_{1}}}{2}\right) .
$$

Proof: The maximum PEP in the spatial domain involves the adjacent symbols of different power levels in the supersymbol constellation and can be expressed as

$$
\begin{aligned}
& \mathcal{P}_{m}\left(s_{m_{i}}^{k} \rightarrow s_{m_{i}}^{l}\right)=\mathcal{P}\left(\left\|r_{i}-s_{m_{i}}^{k}\right\|^{2}>\left\|r_{i}-s_{m_{i}}^{l}\right\|^{2}\right) \\
& \quad=\mathcal{P}\left(P_{1}-2 \mathcal{R}\left\{r_{i}^{*} \sqrt{P_{1}} b_{m_{i}}\right\}>P_{2}-2 \mathcal{R}\left\{r_{i}^{*} \sqrt{P_{2}} b_{m_{i}}\right\}\right),
\end{aligned}
$$

where, using $r_{i}=\sqrt{p_{k_{i}}} b_{m_{i}}+v_{i}$ we get

$$
\begin{aligned}
& \mathcal{P}_{m}\left(s_{m_{i}}^{k} \rightarrow s_{m_{i}}^{l}\right) \\
&= \mathcal{P}\left(P_{1}-2 P_{1}\left|b_{m_{i}}\right|^{2}-2 \mathcal{R}\left\{u_{i}^{*} \sqrt{P_{1}} b_{m_{i}}\right\}\right. \\
&\left.>P_{2}-2 \sqrt{P_{1} P_{2}}\left|b_{m_{i}}\right|^{2}-2 \mathcal{R}\left\{u_{i}^{*} \sqrt{P_{2}} b_{m_{i}}\right\}\right) \\
&= \mathcal{P}\left(2\left(\sqrt{P_{2}}-\sqrt{P_{1}}\right) \mathcal{R}\left\{u_{i}^{*} b_{m_{i}}\right\}>P_{1}+P_{2}-2 \sqrt{P_{1} P_{2}}\right) \\
&= \mathcal{P}\left(-\mathcal{R}\left\{u_{i}^{*} b_{m_{i}}\right\}>\frac{\sqrt{P_{1}}-\sqrt{P_{2}}}{2}\right) .
\end{aligned}
$$

Similarly to the above proof, we have used the fact that $\left|b_{m_{i}}\right|^{2}=1$, and it can be seen that $\psi \hat{=}-\mathcal{R}\left\{u_{i}^{*} b_{m_{i}}\right\} \in$ $\mathcal{N}\left(0, N_{0} / f^{2}\right)$. Accordingly, for the minimum PEP in the spatial constellation we have

$$
\mathcal{P}_{m}\left(s_{m_{i}}^{k} \rightarrow s_{m_{i}}^{l}\right)=\mathcal{P}\left(\psi>\frac{\sqrt{P_{2}}-\sqrt{P_{1}}}{2}\right),
$$

which leads to (22).

The above indicates that the separation between $\left\{P_{1}, P_{2}\right\}$ should be maximized for minimizing the errors in the spatial bits, which are dominated by the distance between the pairs of adjacent symbols having different power levels $d_{s}=$ $\sqrt{P_{1}}-\sqrt{P_{2}}$. We therefore define the spatial function $f_{S}(\alpha)$ that accounts for the dependence of the spatial errors on $\alpha$ as

$$
f_{S}(\alpha) \hat{=} \sqrt{P_{1}}-\sqrt{P_{2}}=\frac{1-\sqrt{\alpha}}{\sqrt{\left(N_{r}-N_{a}\right) \alpha+N_{a}}} .
$$

As regards to the classic modulated symbol errors, it is known that the PSK error probability is given as

$$
\begin{aligned}
\mathcal{P}\left(s_{m_{i}}^{k} \rightarrow s_{n_{i}}^{k}\right) & =\mathcal{P}\left(\left\|r_{i}-s_{m_{i}}^{k}\right\|^{2}>\left\|r_{i}-s_{n_{i}}^{l}\right\|^{2}\right) \\
& =Q\left(f \sqrt{\frac{P_{2}}{N_{0}} \log _{2}(M) \sin \frac{\pi}{M}}\right) .
\end{aligned}
$$

Accordingly, we define the function $f_{M}(\alpha)$ for the dependence of the modulated symbol error on $\alpha$ as

$$
\begin{aligned}
f_{M}(\alpha) & \hat{=} \sqrt{P_{2} \log _{2}(M) \sin \frac{\pi}{M}} \\
& =\sqrt{\log _{2}(M) \sin \frac{\pi}{M} \cdot \frac{\alpha}{\left(N_{r}-N_{a}\right) \alpha+N_{a}}} .
\end{aligned}
$$

The optimization (21) is equivalent to the maximization of the minimum of these functions

$$
\alpha_{\text {opt }}=\arg \max _{\alpha}\left\{\min \left\{f_{S}(\alpha), f_{M}(\alpha)\right\}\right\} .
$$

which is equivalent to selecting the factor $\alpha$ so that the two terms in the minimization become equal, which gives

$$
\alpha_{\text {opt }}=\frac{1}{\left(1+\sqrt{\log _{2}(M) \sin \frac{\pi}{M}}\right)^{2}} .
$$




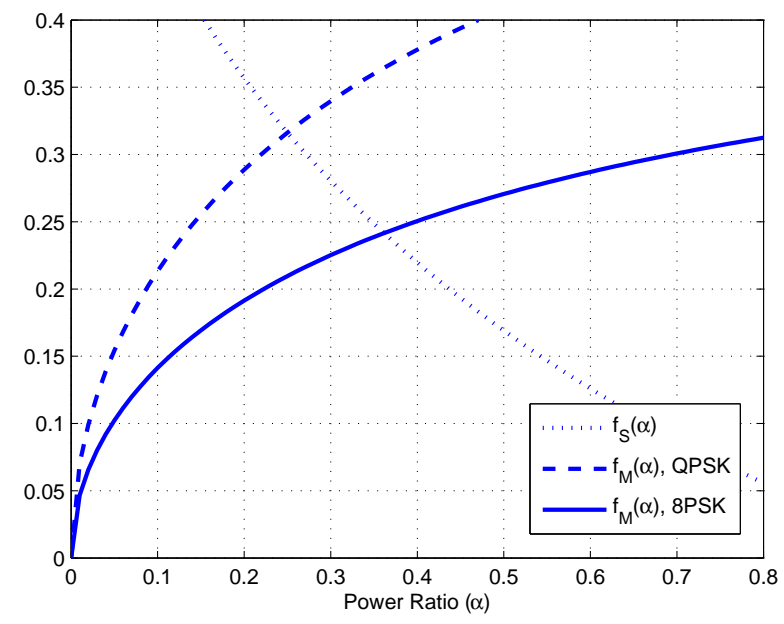

Fig. 2. Theoretical optimization of $\alpha$ for DLT for a $(8 \times 4)$ MIMO, $N_{a}=2$.
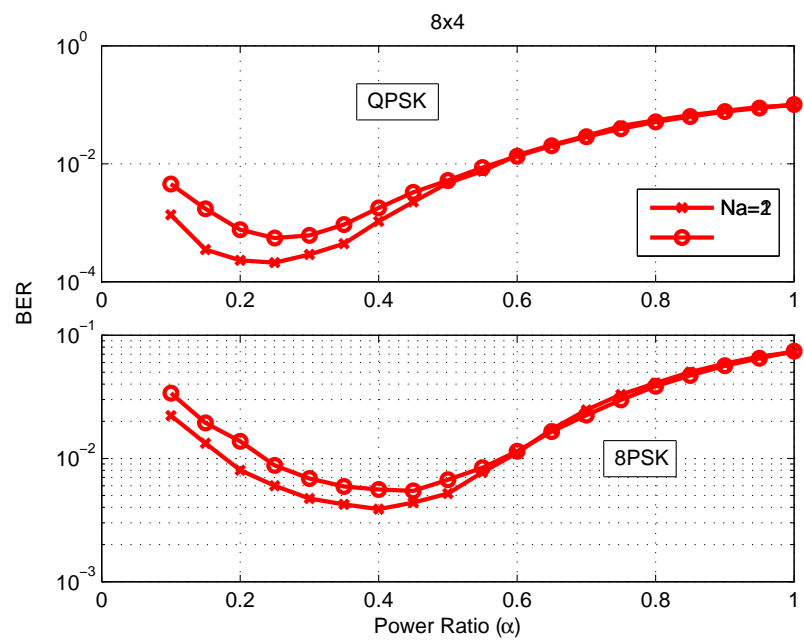

Fig. 3. BER vs. $\alpha$ for a $(8 \times 4)$ MIMO with SMX and DLT, SNR=10dB.

We examine this optimization in Fig. 2 which shows the functions $f_{s}(\alpha), f_{M}(\alpha)$ when increasing the values of $\alpha$ for the example of a $(8 \times 4)$-element DLT system with $N_{a}=$ 2 , for $M=4,8,16$, i.e. QPSK, and 8PSK modulation. The intersections of the lines determine the optimum values of $\alpha$. It will be shown in the following that the theoretically obtained optimal values of $\alpha$ closely match the optimal values obtained by simulation.

\section{Numerical Results}

To evaluate the benefits of the proposed technique, this section presents numerical results based on Monte Carlo simulations of SMX, RSM and the proposed DLT. The channel impulse response is assumed to be perfectly known at the transmitter. Without loss of generality, unless stated otherwise, we assume that the transmit power is restricted to $P=1$. MIMO systems with up to 8 TAs employing QPSK, and 8PSK modulation are explored, albeit it is plausible that the benefits of the proposed technique extend to larger scale systems and higher order modulation.

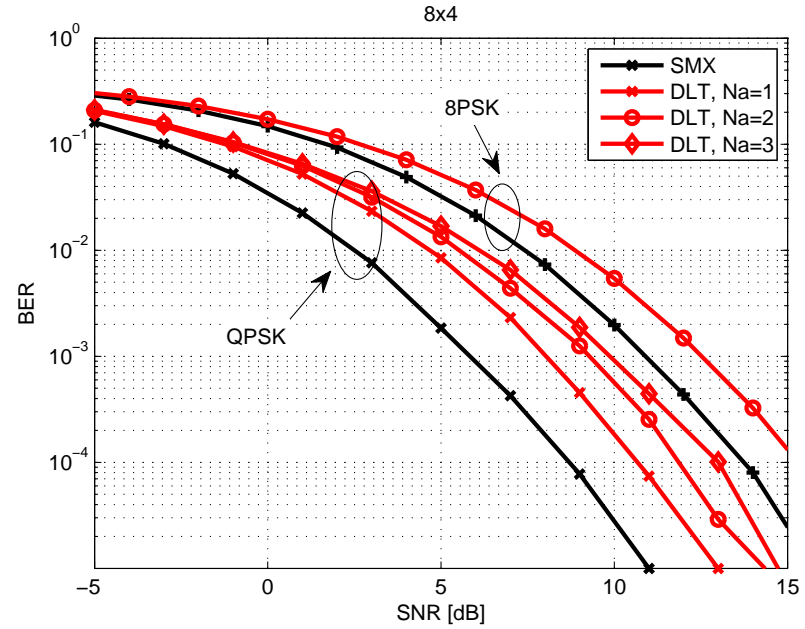

Fig. 4. BER vs. SNR for a $(8 \times 4)$ MIMO with SMX and DLT.

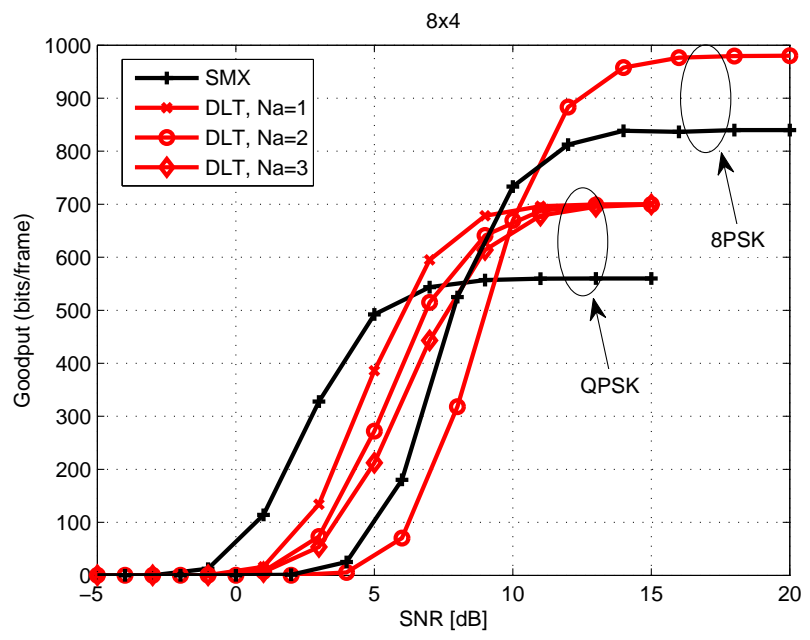

Fig. 5. Goodput vs. SNR for a $(8 \times 4)$ MIMO with SMX and DLT.

In Fig. 3 we show the BER as a function of the power ratio for DLT for the $(8 \times 4)$ MIMO system, where the values of $\alpha$ in the area of 0.25 and 0.4 can be seen to provide the best performance for QPSK and 8PSK respectively. This matches well with the theoretically derived result of Section IV.A and Fig. 2. In Fig. 4 we show the BER with increasing SNR for the proposed DLT, where the black lines for $N_{a}=8$ represent SMX transmission. The curves show results for both QPSK and 8PSK. Clearly, the DLT scheme has inferior BER performance compared to SMX due to the additional spatial streams, but at the benefit of improved BE. The improved BE of DLT is demonstrated in Fig. 5 where the goodput with increasing SNR is depicted for the same $(8 \times 4)$ MIMO scenario. Clearly, DLT provides higher goodput than SMX for sufficiently high SNR values.

\section{CONCLUSions}

A bandwidth efficient transmission scheme was proposed, that combines traditional MIMO SMX with RSM. As opposed to traditional RSM where a subset of antennas carry a spatial 
stream, here we allow all antennas to carry information, by applying RSM on the symbol power level domain. By allowing all antennas to form spatial streams, the proposed scheme has been shown to improve the system's BE and power efficiency compared to both SMX and RSM. Our future work will involve exploring more advanced precoding schemes for the proposed transmission as well as exploring the adaptations of the prosed scheme for robustness channel state information errors.

\section{ACKNOWLEDGMENT}

This work was supported by the Royal Academy of Engineering, UK and the Engineering and Physical Sciences Research Council (EPSRC) project EP/M014150/1.

\section{REFERENCES}

[1] R. Mesleh, H. Haas, S. Sinanovic, C. W. Ahn, and S. Yun, "Spatial modulation," IEEE Trans. Veh. Technol., vol. 57, no. 4, pp. 2228-2241, July 2008.

[2] M. Di Renzo, H. Haas, A. Ghrayeb, S. Sugiura, L. Hanzo, "Spatial Modulation for Generalized MIMO: Challenges, Opportunities, and Implementation," Proceedings of the IEEE, vol. 102, no. 1, pp.56-103, Jan. 2014

[3] M. Di Renzo and H. Haas, "Bit Error Probability of Space Modulation over Nakagami-m Fading: Asymptotic Analysis," IEEE Commun. Lett., vol. 15 , no. 10, pp. 1026-1028, Oct. 2011.

[4] J. Jeganathan, A. Ghrayeb, and L. Szczecinski, "Spatial Modulation: Optimal Detection and Performance Analysis," IEEE Commun. Lett., vol 12, no. 8, pp. 545-547, Aug. 2008.

[5] A. Garcia-Rodriguez and C. Masouros, "Low-complexity compressive sensing detection for spatial modulation in large-scale multiple access channels," IEEE Trans. on commun., vol. 63, no. 7, pp. 2565?2579, July 2015.

[6] P. Yang, Y. Xiao, K. V. S. Hari, A. Chockalingam, S. Sugiura, H. Haas, M. Di Renzo, C. Masouros, Z. Liu, L. Xiao, S. Li, and L. Hanzo, "Single-Carrier Spatial Modulation: A Promising Design for LargeScale Broadband Antenna Systems," IEEE Communications Surveys and Tutorials, submitted

[7] A. Younis, S. Sinanovic, M. Di Renzo, R. Mesleh, H. Haas, "Generalised Sphere Decoding for Spatial Modulation," IEEE Trans. Comms. , vol.61, no.7, pp.2805-2815, July 2013

[8] M. Di Renzo, H. Haas, "On Transmit Diversity for Spatial Modulation MIMO: Impact of Spatial Constellation Diagram and Shaping Filters at the Transmitter," IEEE Trans. Veh. Technol., vol.62, no.6, pp.2507-2531, July 2013

[9] P. Yang, Y. Xiao, B. Zhang, S. Li, M. El-Hajjar, L. Hanzo, "StarQAM Signaling Constellations for Spatial Modulation," IEEE Trans. Veh. Technol., vol.PP, no.99, pp.1,1

[10] S. Sugiura, C. Xu, S. X. Ng, and L. Hanzo, "Reduced-Complexity Coherent versus Non-Coherent QAM-Aided Space-Time Shift Keying," IEEE Trans. Comms., vol.59, no.11, pp.3090-3101, Nov. 2011.

[11] K. Ntontin, M. Di Renzo, A. Perez-Neira and C. Verikoukis, "Adaptive Generalized Space Shift Keying", EURASIP Jour. Wireless Comms and Networking, Vol. 2013, Feb. 2013.

[12] S. Sugiura and L. Hanzo, "On the Joint Optimization of Dispersion Matrices and Constellations for Near-Capacity Irregular Precoded SpaceTime Shift Keying ," IEEE Trans. Wirel. Comms., 2013, vol. 12, no. 1, pp. 380-387, 2013.

[13] M. Maleki, H. Bahrami, S. Beygi, M. Kafashan, N. H. Tran, "Space Modulation with CSI: Constellation Design and Performance Evalution," IEEE Trans. Veh. Technol.,vol. 62, no. 4, pp. 1623-1634, 2013.

[14] X. Guan, Y. Cai, W. Yang, "On the Mutual Information and Precoding for Spatial Modulation with Finite Alphabet," IEEE Wirel. Commun. Lett., vol.2, no.4, pp.383-386, August 2013

[15] J.M. Luna-Rivera, D.U. Campos-Delgado, M.G. Gonzalez-Perez, "Constellation Design for Spatial Modulation," Procedia Technology, vol. 7, pp.71-78, 2013

[16] C. Masouros, "Improving the Diversity of Spatial Modulation in MISO Channels by Phase Alignment," IEEE Commun. Lett., vol.18, no.5, pp.729-732, May 2014
[17] C. Masouros and L. Hanzo, "Constellation-Randomization Achieves Transmit Diversity for Single-RF Spatial Modulation," IEEE Trans. Comms., in press

[18] A. Garcia-Rodriguez, C. Masouros, and L. Hanzo, "Pre-scaling optimization for space shift keying based on semidefinite relaxation," IEEE Transactions on commun., Accepted for publication, 2015.

[19] R. Zhang, L. Yang, L. Hanzo, "Generalised Pre-Coding Aided Spatial Modulation," IEEE Trans. Wirel. Comms. , vol.12, no.11, pp.5434-5443, November 2013

[20] C. B. Peel, B. M. Hochwald, A. L. Swindlehurst, "A Vector-Perturbation Technique for Near-Capacity Multiantenna Multiuser Communication Part I: Channel Inversion and Regularization," IEEE Trans. Coms., vol. 53, no. 1, pp. 195-202, Jan 2005.

[21] C. Masouros, E. Alsusa, "Dynamic Linear Precoding for the Exploitation of Known Interference in MIMO Broadcast Systems," IEEE Trans. on Wireless Comms., vol 8, issue 3, pp. 1396-1404, March 2009.

[22] C. Masouros, M. Sellathurai, T. Ratnarajah, "Vector Perturbation Based on Symbol Scaling for Limited Feedback MIMO Downlinks," IEEE Trans. Sig. Proc., vol. 62, no. 3, pp. 562-571, Feb.1, 2014

[23] C. Masouros, "Correlation Rotation Linear Precoding for MIMO Broadcast Communications," IEEE Trans. on Sig. Proc., vol 59, issue 1, pp. 252-262, Jan 2011

[24] M. Abramowitz and I. A. Stegun, Handbook of Mathematical Functions with Formulas, Graphs, and Mathematical Tables, 1972. 